Article

\title{
Automatic Sun Glint Removal of Multispectral High-Resolution Worldview-2 Imagery for Retrieving Coastal Shallow Water Parameters
}

\author{
Javier Martin *, Francisco Eugenio, Javier Marcello and Anabella Medina \\ Received: 23 September 2015; Accepted: 21 December 2015; Published: 5 January 2016 \\ Academic Editors: Deepak R. Mishra, Richard W. Gould Jr. and Prasad S. Thenkabail \\ Instituto Oceanografía y Cambio Global, Universidad de Las Palmas de G.C., Campus Universitario de Tafira, \\ Las Palmas de G.C. 35017, Spain; feugenio@dsc.ulpgc.es (F.E.); javier.marcello@ulpgc.es (J.M.); \\ allebana@gmail.com (A.M.) \\ * Correspondence: javier.martin.abasolo@gmail.com; Tel.: +34-928-452979; Fax: +34-928-451243
}

\begin{abstract}
Remote sensing of coastal areas requires multispectral satellite images with a high spatial resolution. In this sense, WorldView-2 is a very high resolution satellite, which provides an advanced multispectral sensor with eight narrow bands, allowing the proliferation of new environmental monitoring and mapping applications in shallow coastal ecosystems. These challenges need the accurate determination of the water radiance, which is not often valued compared to other sources such as atmosphere and specular water reflection (sun glint). In this context, the atmospheric correction and the glinting removal have demonstrated to be critical steps in the preprocessing chain of high resolution images. In this work, the Second Simulation of a Satellite Signal in the Solar Spectrum (6S) is used to compensate the atmospheric effects and to compute part of the deglinting algorithm using the modeled direct normalized irradiance. This paper describes a novel automatic deglinting procedure, integrated in the Radiative Transfer Modeling (RTM) inversion of the shallow water environments, which allows computing the water Inherent Optical Properties (IOPs), bathymetry and seafloor albedo contributions. The proposed methodology has demonstrated a proper performance for environmental monitoring in shallow water areas.
\end{abstract}

Keywords: high resolution; Worldview-2; atmospheric $6 \mathrm{~S}$ model; sun glint; shallow water; radiative transfer modeling

\section{Introduction}

Remote sensing is a useful technology to provide timely and up-to-date information for coastal shallow waters environments. The use of multispectral imagery from satellite sensors such as TM (Thematic Mapper) or its new generations, MODIS (Moderate Resolution Imaging Spectroradiometer) or SeaWiFS (Sea-viewing Wide Field-of-view Sensor), has been established for many applications, including the estimation of chlorophyll concentrations, suspended matter, etc. In parallel, very high resolution multispectral imagery sensors started to be launched (Ikonos, Quicbird, etc.) but they recorded energy in only 4 bands. With the advent of WorldView-2 (WV-2), providing better spatial and spectral information, there is an important potential for water quality monitoring, benthic habitat mapping and bathymetry retrieval in littoral zones [1,2].

The ability of WV-2 to change its viewing angle and to supply multispectral information in 8 bands have highlighted the importance of accurately modeling the radiometric and atmospheric effects for shallow-water applications due to the low signal-to-noise ratio available at sensor level on shorter wavelengths. In fact, in such scenarios, the reflectance recorded by satellite sensors is generally low 
and affected by atmospheric absorption and scattering, sensor-target-illumination geometry and sensor calibration [3].

In our previous research, to properly use the multispectral WV-2 data, a basic deglinting algorithm was implemented to remove sea surface effects from high-resolution imagery in shallow-water environments prior to estimating seafloor reflectivity. This procedure was introduced in [4].

In real scenarios, and depending on the area, several portions of the WV-2 multispectral imagery acquired for coastal zones are disappointing, because they are severely contaminated by reflected light (glint) on the crests or slopes of waves, generated by local surface winds [5]. In consequence, the remote water quality properties, bathymetry or mapping of benthic features are seriously impeded by the specular reflection of solar radiation on rough water surfaces [6].

In this work, the crucial atmospheric and sun glint corrections have been improved in order to properly and systematically use the WV-2 multispectral data to improve the monitoring and assessment of water quality and bottom mapping in near-shore environments. Accordingly, the spectral capabilities of WV-2 multispectral imagery are fully exploited for shallow coastal waters applications.

This paper is organized as follows: Section 2 includes the atmospheric multispectral high resolution data preprocessing and the new sea surface correction (deglinting model) for WV-2 images. In this section, the procedure for the Radiative Transfer Equations (RTE) inversion, which integrates the new sun glint algorithm, is presented and analyzed. Section 3 focuses on the main results achieved after the application of the new methodology. Final conclusions are included in Section 4.

\section{Methodology}

The proposed methodology for the processing of multispectral high resolution WV-2 imagery is presented in Figure 1. The different modules that integrate this methodology are:

(i) A radiometric calibration and optimal atmospheric correction model.

(ii) A novel automatic algorithm for sun glint removal based on physical modeling.

(iii) A multichannel physics-based algorithm which integrates the new deglinting technique and the Radiative Transfer Equations for monitoring and mapping coastal shallow water parameters: Inherent Optical Properties, water depth and bottom reflectance.

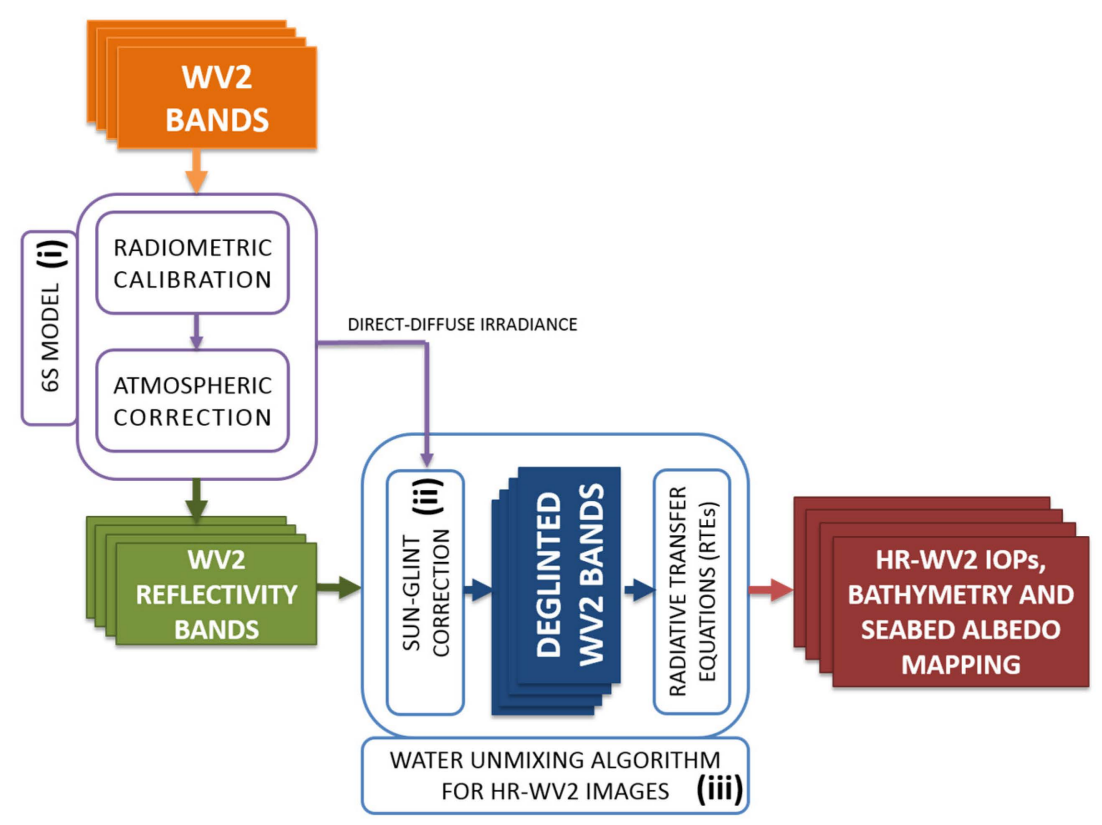

Figure 1. Schematic procedure of the proposed multispectral high resolution WorldView-2 processing chain for shallow coastal waters applications. 
In the following sections, the remote sensing data used in the analysis and the different modules are presented in detail.

\subsection{WorldView-2 Multispectral Imagery and Study Area}

This study is based on Ortho Ready Standard WV-2 imagery, resampled to $2.0 \mathrm{~m}$ spatial resolution, using 11-bit data in eight spectral bands: coastal $(400-450 \mathrm{~nm})$, blue $(450-510 \mathrm{~nm})$, green $(510-580 \mathrm{~nm})$, yellow (585-625 nm), red (630-690 nm), red edge (705-745 nm), NIR1 (770-895 nm), and NIR2 (860-1040 nm). At nadir, the collected nominal ground sample distance is $0.46 \mathrm{~m}$ (panchromatic) and $1.84 \mathrm{~m}$ (multispectral). The nominal swath width is $16.4 \mathrm{~km}$. This implies that WV-2 and the new WV-3 (with spatial resolution of $0.31 \mathrm{~m}$ and $1.24 \mathrm{~m}$ ) satellites provide finer spatial resolution and more spectral information in the visible spectrum than previous satellites, positioning them as the forerunner for oceanographic parameters mapping.

The study area selected is the Canary Islands coastal environment, off the northwest African coast, as shown in Figure 2. In addition, in Figure 2, two high resolution Ortho Ready Standard images acquired by WorldView-2 satellite of two different Canary Islands coastal areas, Maspalomas (Gran Canaria Island) and Corralejo-Lobo Island (Fuerteventura Island), are presented. The Natural Reserve of the Dunes of Maspalomas, which covers an area of approximately 403.9 hectares, consists of three ecosystems: Palmeral, La Charca Lake, and the Dunes. This natural resource extends into the sea creating a perfect habitat for the seagrass beds, which have a great importance in the biodiversity of the Canary Islands coast.

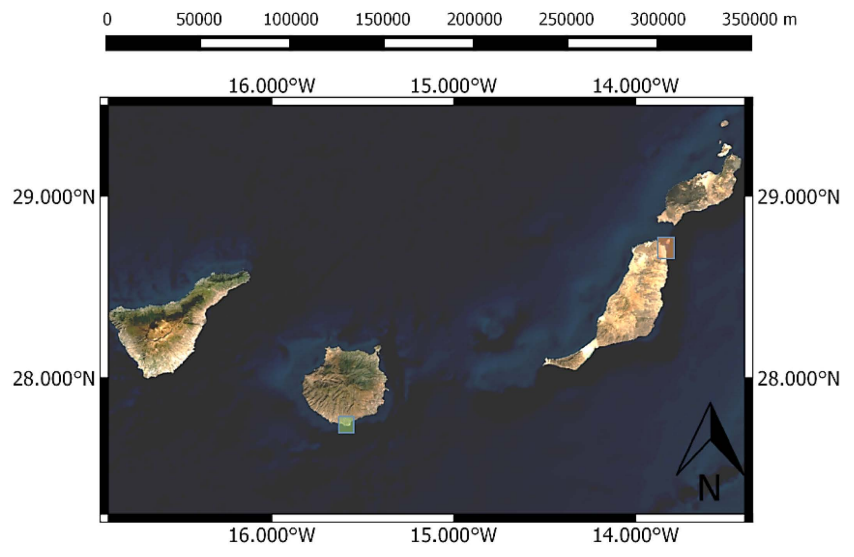

(a)

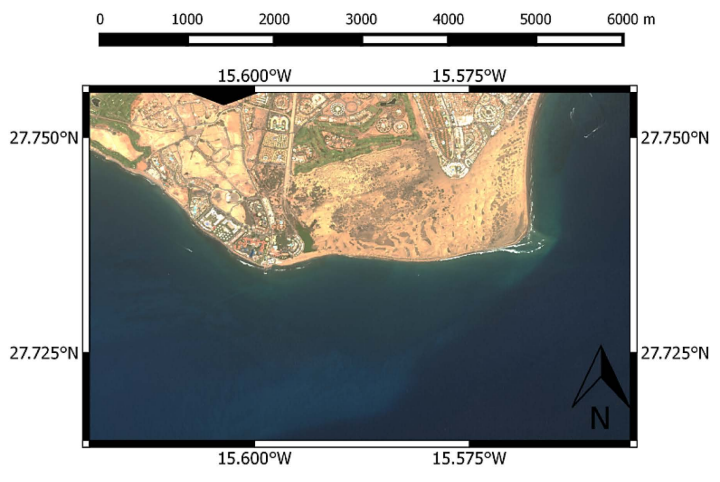

(b)

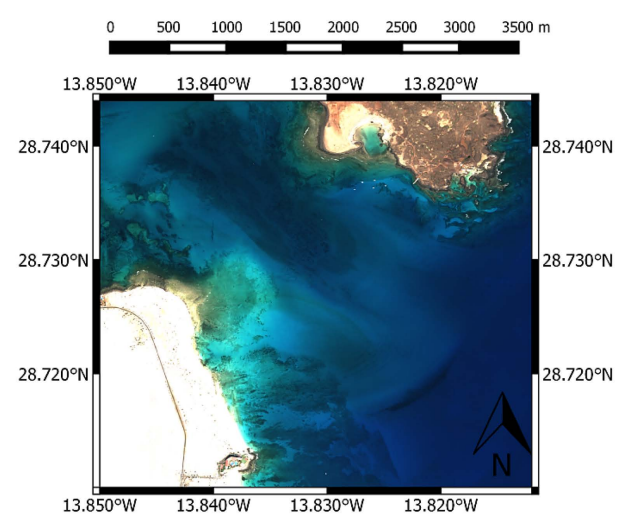

(c)

Figure 2. (a) Location of study area (The Canary Islands); (b,c) WV-2 images of two Canary Islands singular littoral zones: (b) Maspalomas (Gran Canaria Island, 11 August 2013) area; and (c) Corralejo-Lobo Island (Fuerteventura Island, 28 October 2010) area. 
In order to validate the results obtained in the surface reflectance, after the atmospheric correction, a spectroradiometer was used for an in situ sampling. Specifically, the ADS Fieldspec 3 instrument was recording the in situ reflectance above the sea water in the optical and NIR bands. Likewise, the water quality of the Maspalomas coast and La Charca Lake has been sampled in order to obtain phytoplankton, turbidity, and CDOM concentrations.

\subsection{Atmospheric Correction Algorithm}

Atmospheric correction algorithms for multichannel remote sensing from low resolution sensors, for example NASA-MODIS and ESA-MERIS, were designed for retrieving water-leaving radiances over clear deep ocean areas, Case I ocean waters [7], and cannot easily be modified for retrievals over turbid coastal waters. For such turbid coastal environments, the water-leaving radiance may be significantly higher due to the contribution of the suspended material back-scattering and bottom albedo. Hence, applications of the Case I algorithms to satellite imagery acquired over such turbid coastal waters often result in erroneous retrievals and improved atmospheric correction algorithms must be developed for the remote sensing of Case II waters [1,3].

We have proceeded to apply the atmospheric correction of the eight WV-2 bands using the 6S model [8,9]. 6S is a basic RT code used for calculation of lookup tables in the MODIS atmospheric correction algorithm. It can work in both scalar and vector modes. The $6 \mathrm{~S}$ code is based on the method of successive orders of scattering (SOS) approximations and this model predicts the surface reflectance $\rho_{\text {TOC }}$ (Top of Canopy: TOC) from the reflectance $\rho_{\text {TOA }}$ obtained at the Top of Atmosphere (TOA), using information about the environment surface reflectance (clear water) and atmospheric conditions.

Furthermore, 6S estimates the percentage of direct-diffuse-environmental normalized irradiance at ground level. This diffuse irradiance, generated mainly by the Rayleigh's scattering, has more importance in the blue spectral channels. In order to check the proper functioning of the selected $6 \mathrm{~S}$ algorithm, ground-based spectral data, nearly coincident with a WV-2 satellite over flight, was used [4].

\subsection{Sun Glint Multispectral Data Correction Algorithm}

Specular reflection of direct sun light on water surfaces is a serious inconvenience for the retrieval of water quality, bathymetry and seafloor mapping in shallow-water environments. In the last decades, glint correction methods have been developed for open ocean imaging and higher resolution coastal applications $[5,10]$. These techniques are based on the assumption the near-infrared (NIR) radiation of deep water zones is negligible and, in consequence, NIR bands give an indication of the amount of glint in the received signal. To correct it, the spectrum for a deep water part of the image is manually selected and used to establish the relationship between the NIR and glint radiances, as reviewed in [5].

In our previous study [4], we followed the common procedure suggested by [5]. As indicated, one or more regions of the image are selected where a range of sun glint is evident, but where the underlying spectral brightness would be expected to be consistent, i.e., areas of deep and clear water. For each visible band, all the selected pixels are included in a linear regression $\left(b_{i}\right)$ of NIR brightness against the visible band brightness, as follows,

$$
\begin{gathered}
R_{r s}(\lambda)=\frac{\rho_{T O C}(\lambda)}{\pi} s r^{-1} \\
R_{r s}^{d}(\lambda)=R_{r s}(\lambda)-b_{\lambda} \times\left[R_{r s}(N I R)-\min \left(R_{r s}(N I R)\right)\right] \\
R_{r s}^{d}(\lambda)=\frac{\alpha\left(\theta_{s}\right) \times r_{r s}(\lambda)}{1-\beta\left(\theta_{s}\right) \times r_{r s}(\lambda)}
\end{gathered}
$$

where $\rho_{\text {TOC }}(\lambda)$ is the surface reflectance; $R_{r S}(\lambda)$ is the remote sensing reflectance; $R_{r s}^{d}(\lambda)$ is the deglinted remote sensing reflectance; $\min \left(R_{r s}(N I R)\right)$ is the minima NIR reflectance of the areas; $r_{r s}$ is the subsurface remote sensing reflectance; $\alpha\left(\theta_{s}\right)$ and $\beta\left(\theta_{s}\right)$ are coefficients, based on Hydrolight 
simulations [10], which depend on the zenithal angle [11], $\alpha\left(\theta_{\mathcal{S}}\right)=\left(0^{\circ}=0.5236,30^{\circ}=0.5169\right.$, $\left.60^{\circ}=0.4933\right), \beta\left(\theta_{\mathcal{S}}\right)=\left(0^{\circ}=2.1941,30^{\circ}=2.3001,60^{\circ}=2.6796\right)$.

In Figure 3, an example of linear fitting of the WV-2 reflectance bands R3 and R7 is presented. This example corresponds with a suitable scenario of wind and sea conditions (Maspalomas area, May 2012). We can observe a good fitting result with $R^{2}=0.9573$, obtaining a slope parameter $b=0.9373$.

Table 1 presents the linear fitting results of the visible channels with the corresponding NIR group bands. Note that it is important to use the correct subgroup sensor bands (MS1 and MS2) with its corresponding NIR channel since the WV-2 subgroup sensor bands are not temporally correlated, otherwise very low fitting quality will be obtained [12].

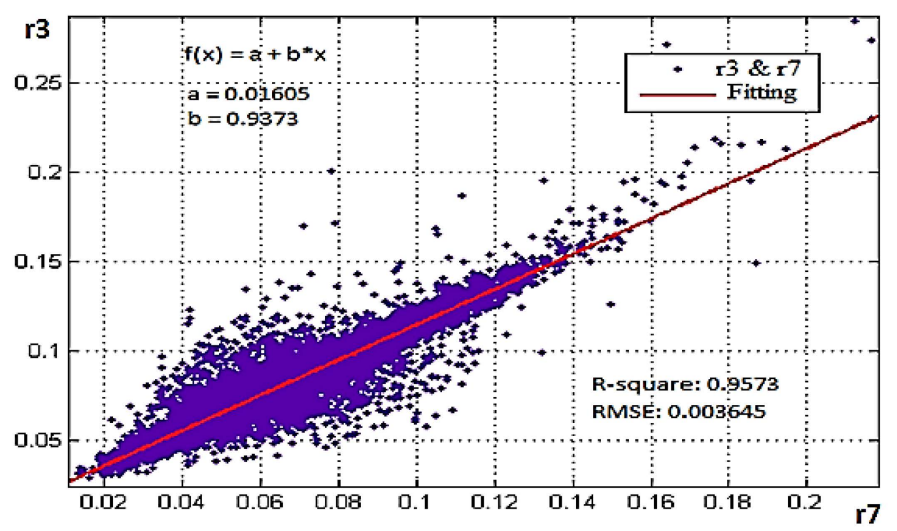

Figure 3. Linear fitting of the green (R3) and NIR1 (R7) WV-2 bands.

Table 1. Linear NIR and visible band fitting results.

\begin{tabular}{ccccccc}
\hline & $\boldsymbol{R} 7$ & $\boldsymbol{R} 8$ & $\boldsymbol{R}^{\mathbf{2}}$ & $\boldsymbol{a}$ & $\boldsymbol{b}$ & $\boldsymbol{m i n}\left(\rho_{\text {TOC }}(\mathrm{NIR})\right)$ \\
\hline$R 1$ & & $\mathrm{X}$ & 0.8513 & 0.04074 & 0.7854 & 0.0064 \\
$R 2$ & $\mathrm{X}$ & & 0.9561 & 0.03918 & 0.8943 & 0.0082 \\
$R 3$ & $\mathrm{X}$ & & 0.9373 & 0.01605 & 0.9673 & 0.0082 \\
$R 4$ & & $\mathrm{X}$ & 0.8836 & 0.01434 & 0.9848 & 0.0064 \\
$R 5$ & $\mathrm{X}$ & & 0.9582 & 0.006162 & 1.003 & 0.0082 \\
$R 6$ & & $\mathrm{X}$ & 0.7946 & 0.004212 & 1.007 & 0.0064 \\
\hline
\end{tabular}

We can appreciate how the $a_{i}$ parameter corresponds to the quite clear ocean water reflectance (being a greatly reduced reflectance), when the glint contribution is null. The other important fact that we can observe is how the $b_{i}$ parameter tends to 1 when the bands move towards the NIR band wavelength.

In this context, we propose a new method based on physical principles for the removal of sea surface effects from high-resolution imagery. The new deglinting algorithm attempts to obtain a physical relation to the slope $b_{i}$, which is calculated from an empirical way in the traditional algorithm.

Observing the simplified schematic diagram [13], which shows routes by which light can reach a remote sensing detector (see Figure 4), we can model the physical phenomena underlying in the slope parameter $b_{i}$. Therefore, the simplified model is:

- A-route is the down directed sun beam.

- B-route is the scattering of the atmosphere to the water surface and then reflected to the satellite (sky-glint).

- C-route is the water-leaving reflectance transmitted through the atmosphere and the air-water surface.

- D-route is the specular reflection of the water surface (sun glint).

- $\quad$ E-route is the single or multiple backscattering of the atmosphere (Rayleigh and aerosol). 
Once corrected the atmospheric disturbances (absorption and E-route component), and following the assumption that in the NIR band the water absorbs all radiation, the reflectivity of this band corresponds only to the specular contribution of surface brightness. In rough water conditions, specular reflectivity is mainly due to the direct irradiation, while diffuse irradiation represents a minor contribution. The diffuse irradiation is distributed equally in all directions (due to the Rayleigh scattering phase function [14]), producing an average brightness of all the contributions of the light scattered by the atmosphere with different angles (B-route component), making it fundamentally independent of the sea state [15].

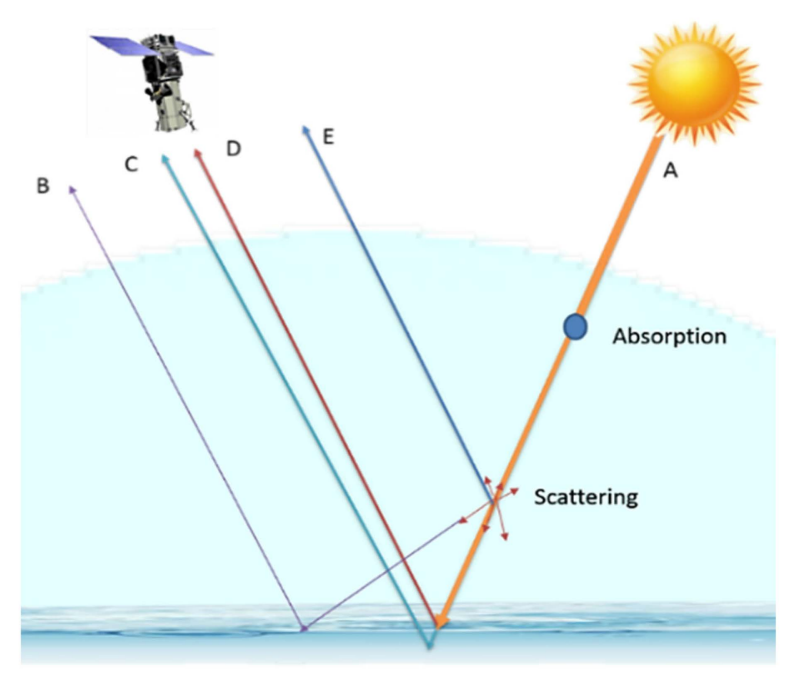

Figure 4. Simplified schematic diagram showing routes by which light reaches a remote sensing detector.

In order to measure the specular reflected reflectance compared to the radiation transmitted by the water surface, the Fresnel formula is often used $[5,13]$. This formula depends on the wavelength of the light used to obtain the refractive index $(n)$ of the sea water, and the incident angle of the light with respect to the surface $(\omega)$ :

$$
\begin{gathered}
\rho_{\omega}^{\text {Fresnel }}=\frac{1}{2} \times\left[\left(\frac{\sin \left(\omega-\omega^{\prime}\right)}{\sin \left(\omega+\omega^{\prime}\right)}\right)^{2}+\left(\frac{\tan \left(\omega-\omega^{\prime}\right)}{\tan \left(\omega+\omega^{\prime}\right)}\right)^{2}\right] \\
\omega^{\prime}=\operatorname{asin}\left(\frac{\sin (\omega)}{n}\right)
\end{gathered}
$$

Considering that a $2 \times 2 \mathrm{~m}^{2}$ pixel contains some facets with different incident angles obtained in small time windows, we can use the statistical approach, used in low resolution images, to present the problem $[13,16]$,

$$
\rho_{\lambda}^{\text {sunG }}=\frac{L_{\lambda}}{E_{\lambda}}=\frac{\rho_{\lambda}^{\text {Fresnel }}(\omega) \times P\left(\theta_{s}, \theta_{v}, \varphi_{s}, \varphi_{v}\right)}{4 \cos ^{4} \beta \cos \theta_{v}}
$$

where $\rho_{\lambda}^{\operatorname{sun} G}$ is the sun glint reflectance at the surface, $L_{\lambda}$ is the radiance reflected in the surface towards the sensor, $E_{\lambda}$ is the irradiance incident on the surface, $\rho_{\lambda}^{\text {Fresnel }}(\omega)$ is the Fresnel reflectance with respect the incident angle $(\omega), P\left(\theta_{v}, \theta_{s}, \varphi_{s}, \varphi_{v}\right)$ is the provability of specular surface with respect the solar and satellite zenith angles $\left(\theta_{s}, \theta_{v}\right)$ and the solar and satellite azimuth angles $\left(\varphi_{s}, \varphi_{v}\right), \beta$ is the slope inclination angle relative to the flat surface.

Figure 5 shows the specular reflection geometry on a flat surface and the related angles. 


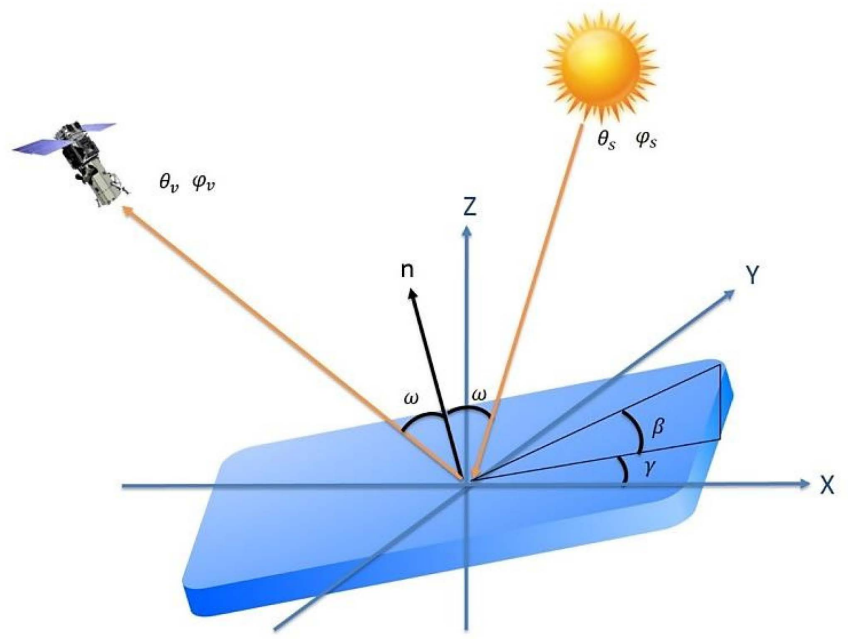

Figure 5. Specular reflection geometry on a flat surface.

Contrary to the specular sun glint, sky glint is weakly dependent on the sea state, allowing to model this reflectance by a parameter which depends on the solar zenith angle and the wind speed [17],

$$
\rho_{\lambda}^{s k y G}=\rho_{\lambda}^{s k y G-c o r r}\left(\theta_{s}, U^{w i n d}\right) \times L_{\lambda}^{s k y}
$$

where $\rho_{\lambda}^{s k y G}$ is the sky glint reflectance at the surface, $\rho_{\lambda}^{\text {skyG-corr }}\left(\theta_{s}, U^{\text {wind }}\right)$ is the sky glint correction coefficient relative to the sun zenith angle and the wind speed $\left(U^{\text {wind }}\right), L_{\lambda}^{\text {sky }}$ is the sky leaving radiance.

Using this approach, and dividing the contribution of the sun glint by the normalized irradiance parameters, we can obtain the following relations between the sea reflectance and the normalize irradiance for the optical and NIR bands,

$$
\begin{gathered}
\rho_{\lambda}=E_{\lambda}^{N D i r} \times \rho_{\lambda}^{s u n G}+E_{\lambda}^{N D i f} \times \rho_{\lambda}^{s k y G}+\rho_{\lambda}^{w} \\
\rho_{N I R}=E_{N I R}^{N D i r} \times \rho_{N I R}^{s u n G}+E_{N I R}^{N D i f} \times \rho_{N I R}^{s k y G}
\end{gathered}
$$

where $E_{\lambda}^{N D i r}$ is the normalized direct irradiance, $E_{\lambda}^{N D i f}$ is the normalized diffuse irradiance, and $\rho_{\lambda}^{w}$ is the deglinted water-leaving reflectance.

By using two in-layer points of the fitted line (Figure 3), we can obtain the following relationship for the slope $b_{\lambda}$ parameter,

$$
\begin{aligned}
& b_{\lambda}=\frac{E_{\lambda}^{N D i r} \times \rho_{\lambda}^{\text {sunG }}(\max )+E_{\lambda}^{N D i f} \times \rho_{\lambda}^{s k y G}+\rho_{\lambda}^{w w}-\left(E_{\lambda}^{N D i r} \times \rho_{\lambda}^{\text {sunG }}(\min )+E_{\lambda}^{N D i f} \times \rho_{\lambda}^{s k y G}+\rho_{\lambda}^{w}\right)}{E_{N I R}^{N D i r} \times \rho_{N I R}^{\text {sunG }}(\max )+E_{N I R}^{N D i f} \times \rho_{N I R}^{\text {sky } G}-\left(E_{N I R}^{N D i r} \times \rho_{N I R}^{\text {sunG }}(\min )+E_{N I R}^{N D i f} \times \rho_{N I R}^{\text {skyG }}\right)} \\
& b_{\lambda}=\frac{E_{\lambda}^{N D i r}}{E_{N I R}^{N D i r}} \times \frac{\left(\rho_{\lambda}^{\text {sunG }}(\max )-\rho_{\lambda}^{\text {sunG }}(\min )\right)}{\left(\rho_{\text {NIR }}^{\text {sunG }}(\max )-\rho_{\text {NIR }}^{\text {sunG }}(\min )\right)} \approx \frac{E_{\lambda}^{\text {NDir }}}{E_{N I R}^{\text {NDir }}}
\end{aligned}
$$

where $\rho^{\operatorname{sun} G}(\max )$ is the maximum sun glint reflectance of the two in-layer points due to the higher level of faces oriented with specular angle, $\rho^{\text {sunG }}(\mathrm{min})$ is the minimum sun glint reflectance due to the lower level of faces oriented with specular angle. Note how $\rho_{\lambda}^{\text {sunG }} \approx \rho_{N I R}^{\text {sunG }}$ due to Fresnel reflectance of the different optical wavelengths vary only slightly since the reflectance index is very similar, being the same assumption that is made in the traditional method.

Next, in Table 2 the direct and diffuse normalized irradiance parameters, produced with the $6 \mathrm{~S}$, are shown. In the same way, the direct irradiances ratio between the bands to correct and the corresponding NIR are presented. 
Table 2. Direct-Diffuse irradiance comparison.

\begin{tabular}{ccccccccc}
\hline & $\boldsymbol{R} \mathbf{1}$ & $\boldsymbol{R 2}$ & $\boldsymbol{R 3}$ & $\boldsymbol{R 4}$ & $\boldsymbol{R 5}$ & $\boldsymbol{R 6}$ & $\boldsymbol{R 7}$ & $\boldsymbol{R} 8$ \\
\hline $\boldsymbol{E}_{\text {band }}^{\text {NDir }}$ & 0.214 & 0.158 & 0.114 & 0.091 & 0.077 & 0.067 & 0.058 & 0.052 \\
\hline $\boldsymbol{E}_{\text {band }}^{\text {NDif }}$ & 0.786 & 0.842 & 0.886 & 0.909 & 0.923 & 0.933 & 0.942 & 0.948 \\
\hline $\boldsymbol{E}_{\lambda}^{\text {NDir }}$ & 0.829 & 0.893 & 0.941 & 0.958 & 0.979 & 0.984 & - & - \\
\hline $\boldsymbol{E}_{\text {NIR }}^{\text {NDir }}$ & & & & & & & & \\
\hline
\end{tabular}

Figure 6 displays the comparison chart of the results of slope $b_{i}$, obtained through linear fitting, versus the ratio of $6 \mathrm{~S}$ direct irradiance parameters.

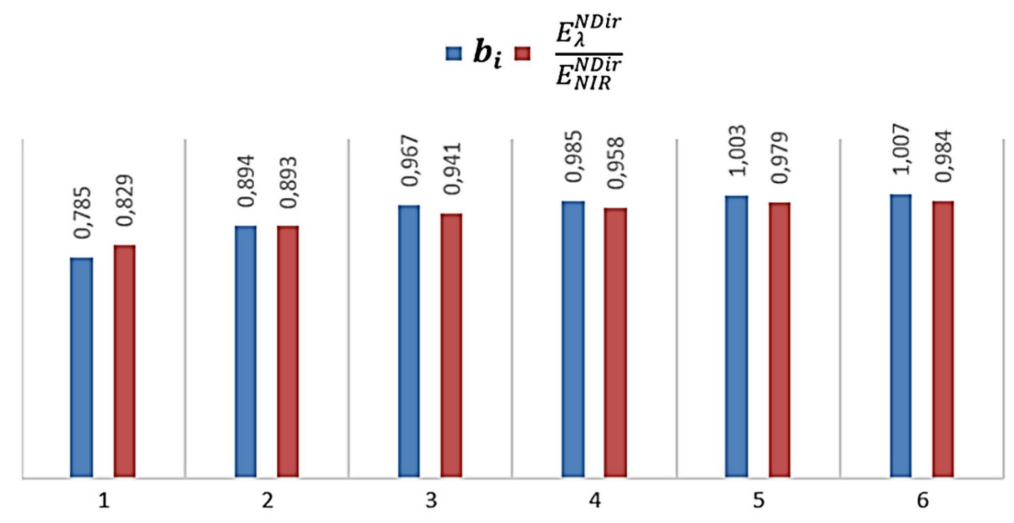

Figure 6. Slope $b_{i}$, obtained by linear fitting (blue); and optical-band/NIR direct irradiance ratio (red).

It can be observed that results are quite similar, although the biggest difference is located in the blue coastal band, where the error in the $6 \mathrm{~S}$ aerosol modeling has a bigger impact on the result of direct-diffuse normalized irradiance computation.

Thus, Equation (9) provides the new deglinting method based on the direct-irradiance ratio. The minimum NIR reflectance has been removed from the equation, since this value is more connected with residual atmospheric aerosol reflectance values than real NIR seawater contribution. The slope has been replaced by the direct-irradiance ratio, providing a fully automatic procedure avoiding the dependence of the selected areas for calculation of the slope $b_{i}$, where such areas must be deep and without turbidity. This is not always possible in shallow coastal environments and in inland waters, allowing, as well, to obtain a fully automatic deglinting algorithm,

$$
R_{r s}^{d}(\lambda)=R_{r s}(\lambda)-\frac{E_{\lambda}^{N D i r}}{E_{N I R}^{N D i r}} \times R_{r s}(N I R)
$$

\subsection{Deglinting Algorithm Integrated into the Radiative Transfer Modeling (RTM)}

Coastal regions are one of the environments with more biodiversity around the globe. Therefore, the mapping of these areas is critical to preserve such shallow coastal ecosystems. Water leaving radiance emanating from shallow coastal waters contains information about the water optical properties, depth and bottom albedo. As previously described, the removal of sun glint is essential to the marine radiative modeling. The algorithms based on eliminating sun glint through the NIR band have a weak point in common: the reflectivity of the water in this channel is not always negligible in very shallow environments and in water with high concentrations of turbidity. These assumptions are very common in coastal and in inland waters, causing significant errors even without the presence of sun glint. To overcome this problem, this paper proposes to integrate the sun glint removal algorithm 
in the radiative transfer model with the goal of modeling the contribution of the NIR reflectance of coastal waters, which will allow us to eliminate this contribution of the specular NIR reflectance.

The forward radiative transfer problem can be solved numerically. Nevertheless, the inverse problem is more challenging. In our context, an efficient multichannel physics-based algorithm has been implemented, capable of solving through optimization the RTE (Radiative Transfer Equations) of coastal waters by integrating the new automatic deglinting algorithm. This new procedure has yielded good results using the radiative model to compute water quality, as it considers the physical phenomena of water absorption-backscattering $\left(k_{d}\right)$ and the relationship between the seafloor albedo, its depth and the water Inherent Optical Properties (IOPs), since they are a function of water quality and the chlorophyll-a, turbidity, and CDOM concentrations. In consequence, the RTE allows us to model the reflectivity and it can be expressed by $[1,4,18,19]$,

$$
r_{r s}^{m} \approx r_{r s}^{d p}\left(1-e^{-\left[\frac{1}{\cos \left(\theta_{\mathrm{s}}^{-}\right)}+\frac{D_{u}^{c}}{\cos \left(\theta_{\mathrm{v}}^{-}\right)}\right] k_{d} z}\right)+\frac{\rho}{\pi} e^{-\left[\frac{1}{\cos \left(\theta_{\mathrm{s}}^{-}\right)}+\frac{D_{u}^{b}}{\cos \left(\theta_{\mathrm{v}}^{-}\right)}\right] k_{d} z}
$$

where $r_{r s}^{m}$ is the modeled subsurface reflectivity; $r_{r s}^{d p}$ is the subsurface reflectivity of the deep water; $\rho$ is the seafloor albedo; $\theta_{s}^{-}$is the subsurface solar zenith angle; $\theta_{v}^{-}$is the subsurface view angle; $D_{u}^{c}$ and $D_{u}^{b}$ are the optical path-elongation factors for scattered photons from the water column and the bottom; $k_{d}$ is the diffuse attenuation coefficient; and $z$ is the depth [19-22].

In order to model the NIR above surface reflectance parameter $\left(R_{r s}^{m}(N I R)\right)$ we have used the RTE expression (Equation (10)) above the surface (Equation (3)). $R_{r s}^{m}(N I R)$ is integrated in the deglinting expression (Equation (9)) in the optimization procedure, which allows to remove the NIR water reflectance amount in very shallows waters (usually less than $2 \mathrm{~m}$ deep), and high suspended solid content, as it is commonly found in very near shore areas,

$$
R_{r S}^{d}(\lambda)=R_{r s}(\lambda)-\frac{E_{\lambda}^{N D i r}}{E_{N I R}^{N D i r}} \times\left(R_{r s}(N I R)-R_{r s}^{m}(N I R)\right)
$$

In the implementation of the gradient optimization technique, Levenberg-Marquardt algorithm (LMA) [23] for solving the nonlinear cost function was employed (Equation (10)). The cost function expression is defined in Equation (10), where the modeled reflectances are compared with the deglinted above surface reflectance, trying to minimize the $\varepsilon_{\min }$ error. The initialization of the $z$ parameter has been performed using the ratio algorithm result [24], for better and faster optimization convergence,

$$
\varepsilon_{\min }=L M A\left(\sum_{c h_{1}}^{c h_{6}}\left\{\frac{R_{r s}^{m}(c h)-R_{r s}^{d}(c h)}{R_{r s}^{d}(c h)}\right\}^{2}\right)
$$

The use of the first six optical plus NIR bands, increases the spectral information, properly removing the sun glint in near shore environments. This fact lets the reflectance unmixing of the water IOPs, the depth and seafloor, which allows more precise water quality calculation even with large matter concentration, and at short depths with non-negligible bottom NIR reflectance influence.

\section{Results and Discussion}

In this section, a summary of the main results achieved in the study areas using the WV-2 imagery are presented. In particular, images with different degrees of sun glint, turbidity and bathymetry are considered to highlight the benefits of our proposed method to correct them and to generate value-added products.

The result of the proposed deglinting algorithm (Equation (9)) is shown in Figure 7. This example presents an atmospherically corrected WV-2 multispectral imagery from Maspalomas area with 
pronounced glint over the sea surface, as shown in Figure 7a. After the glint was removed, subsurface features became pronounced, as shown in Figure 7b.

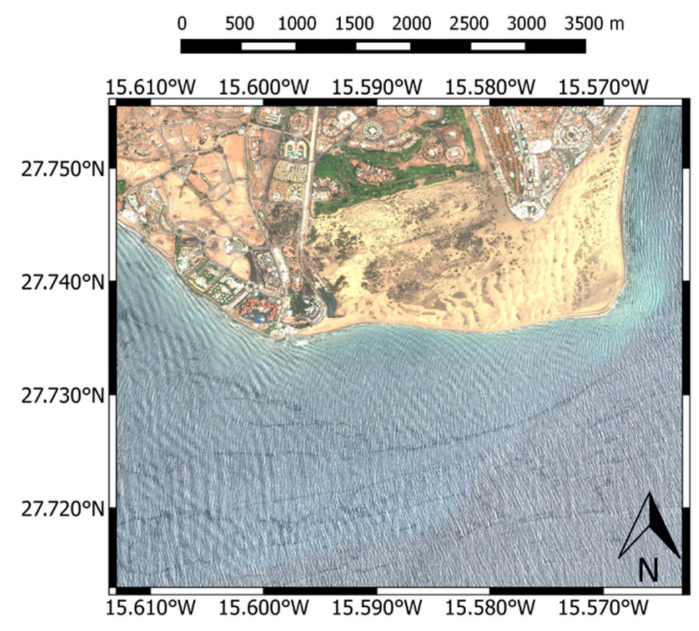

(a)

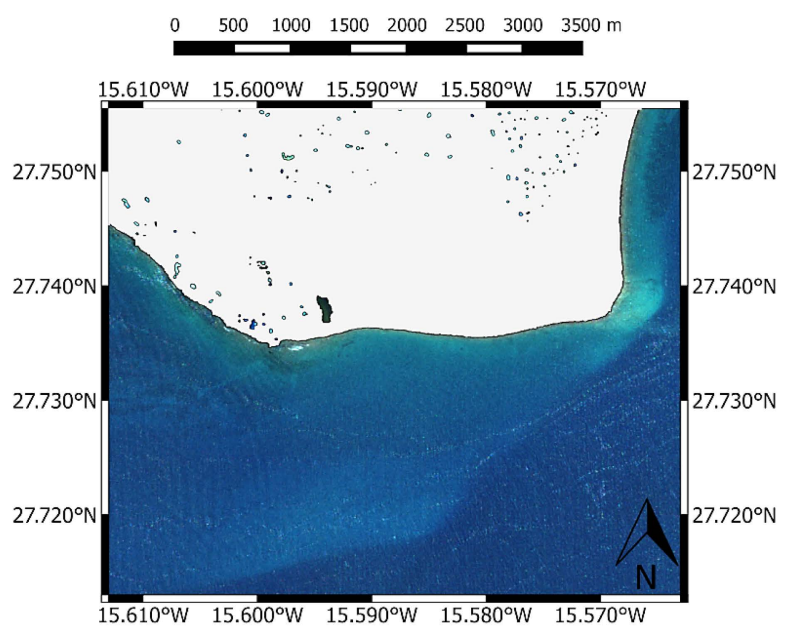

(b)

Figure 7. (a) WV-2 image of Maspalomas before deglinting removal; (b) image after deglinting.

To address the water quality monitoring, two essential parameters have been studied. Firstly, the photosynthetic pigment chlorophyll-a (Chl-a) is a key indicator of phytoplankton biomass, commonly used to assess the eutrophic status of water bodies. Secondly, the diffuse attenuation coefficient $\left(k_{d}\right)$ is an important water property related to light penetration and availability in aquatic systems.

Figure 8 shows the Chl- $a$ concentration in the Maspalomas area (inner lake) for August 2013 image. In Figure $8 \mathrm{a}$ the three in situ measurements acquired at an early date $(\mathrm{P} 1=37.8, \mathrm{P} 2=40.3$, $\mathrm{P} 3=33.0 \mathrm{mg} / \mathrm{m}^{3}$ ) can be observed. Figure $8 \mathrm{~b}$ represents the Chl- $a$ concentration using the classic deglinting + RTE algorithm. On the other hand, the Chl- $a$ concentration using the new automatic deglinting algorithm integrated in the RTE model is shown in Figure 8c. Comparing both results with in situ data, a more accurate remote measurement with the new approach can be observed. It can be seen in Figure $8 \mathrm{~b}$ as the high turbidity of lake water increases the reflectance in the NIR band to the point of introducing significant errors in the estimation after subtracting the NIR reflectance in the classic sun glint removal algorithm.

The result of $k_{d}(490)$ coefficient map, using the new deglinting algorithm integrated in the RTE model, of Corralejo-Lobo Island (Fuerteventura Island) is shown in Figure 9a. We can observe in the Figure 9a more details in the information of the water quality and the local-scale structures. Modeling the non-negligible reflectivity in the NIR band improves the sensitivity to detect the water quality in shallow waters. A more accurate determination of water quality allows detecting local structures which determine the direction of the currents and the functioning of the coastal dynamics [25]. On the other hand, results of seafloor albedo are presented in Figure 9b, after using the new deglinting algorithm integrated in the RTE model, for the selected Corralejo-Lobo Island littoral zones. The existence of the NIR reflectivity, from the seabed albedo, which is subtracted in the other bands in the classic deglinting algorithm, results in a loss of quality in the retrieving of the seabed albedo. In Figure $9 \mathrm{~b}$ we can appreciate how after modeling the NIR reflectance band, this non-negligible contribution of reflectance, can be removed from the correction to yield higher albedos in the very low depths.

Finally, Maspalomas coastal shallow water bathymetry (Southeast area) is shown in Figure 10. Figure 10a presents the sonar bathymetry of the Maspalomas shore, while in Figure 10b the bathymetry using the new deglinting algorithm, integrated in the RTE model is shown. The enhanced capacity provided by the WV-2 imagery, has an important precision in remote sensing water-depth retrievals. 
These results suggest excellent retrieval of bathymetry from high resolution multispectral remote sensing imagery. These results are consistent with expectations in very shallow areas. Note that differences between the sonar bathymetry, Figure 10a, and remote bathymetry, Figure 10b, may be due to the large time difference in the data acquisition and due to the sandy bottom of this coast, which can have some variations depending on the currents. Where the variation in the coastline is an indicator of this fact.

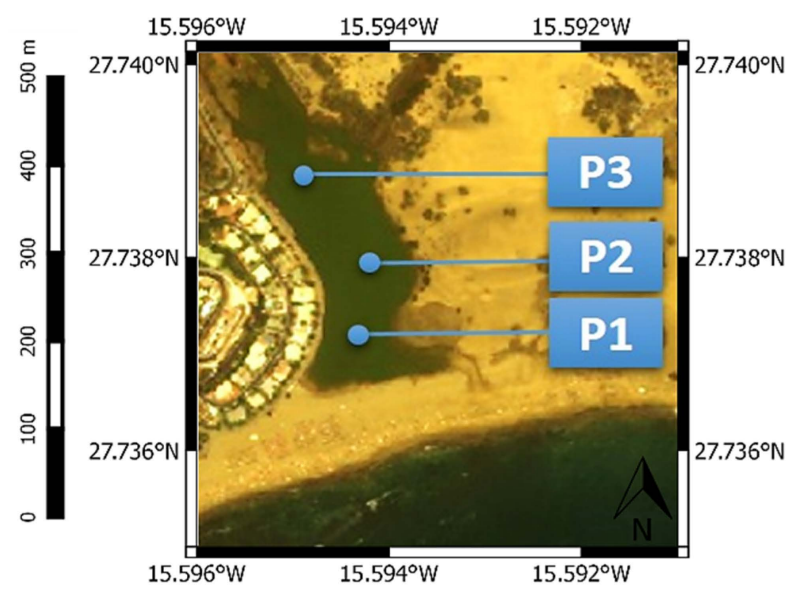

(a)

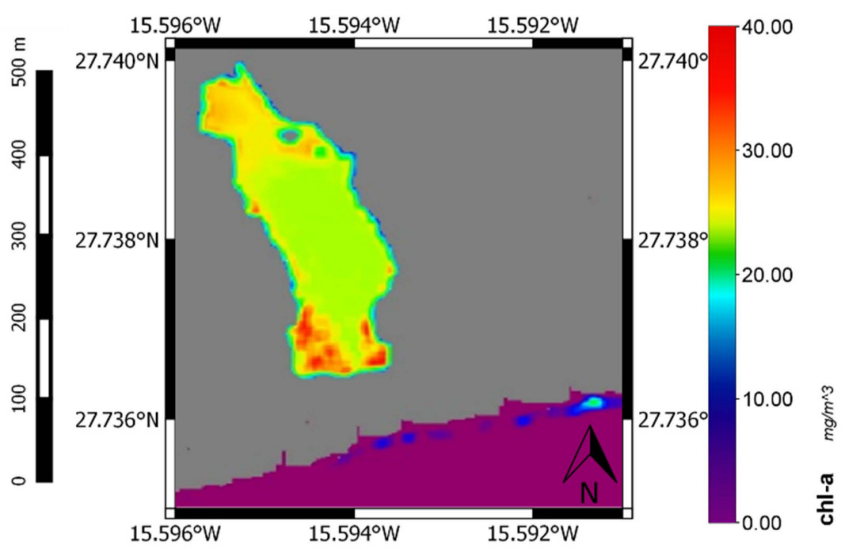

(b)

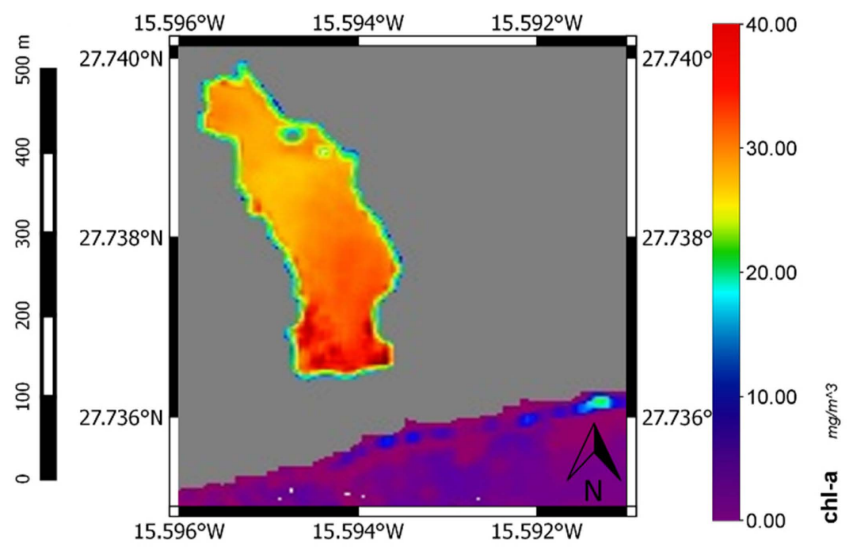

(c)

Figure 8. (a) In situ Chl-a samples in Maspalomas inner lake; (b) Chl-a concentration map by using classical + RTE deglinting algorithm; (c) Chl-a concentration map by using developed deglinting algorithm. 


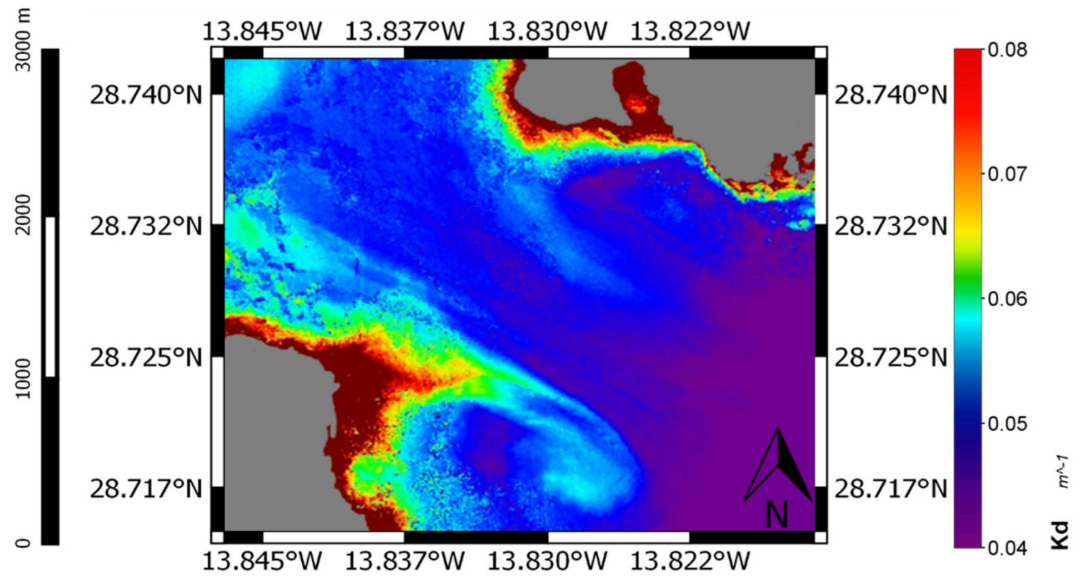

(a)

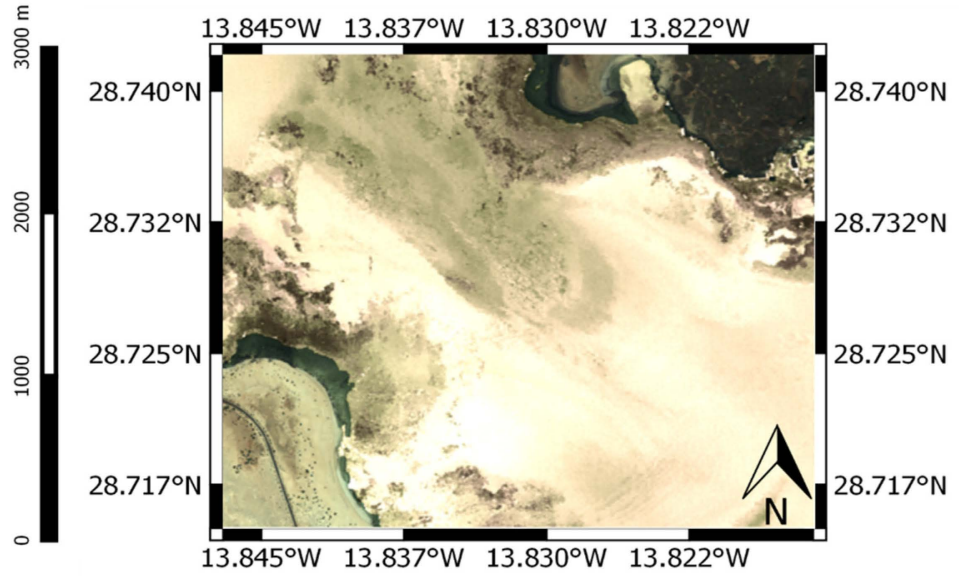

(b)

Figure 9. Corralejo and the Lobo Island (Fuerteventura Island), biosphere reserve and natural protected area: (a) $k_{d}(490)$ coefficient map; and (b) color composite of the seafloor albedo obtained using the new deglinting integrated in the RTE inversion algorithm.

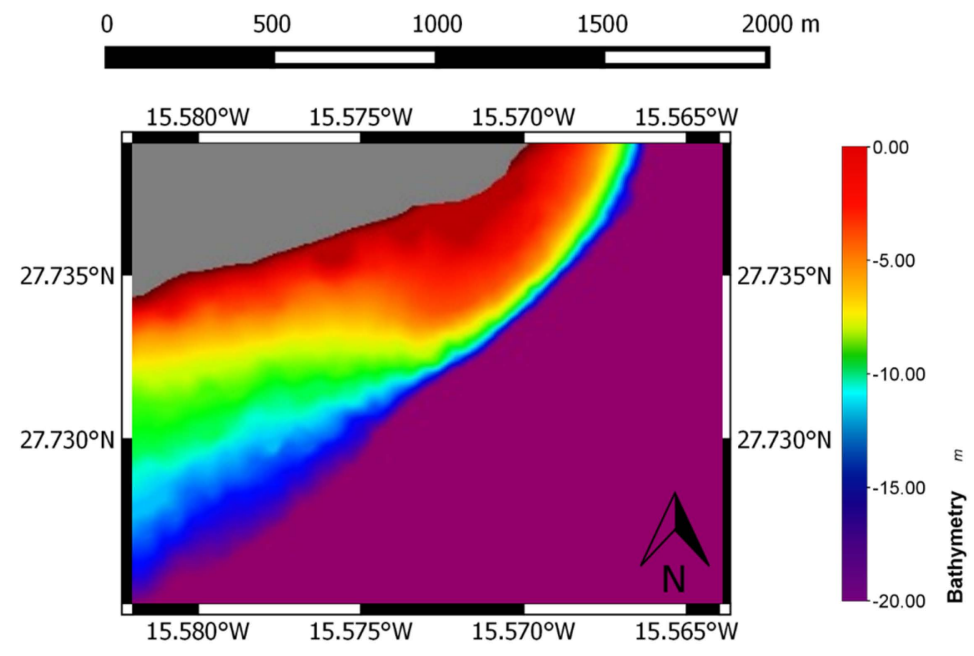

(a)

Figure 10. Cont. 


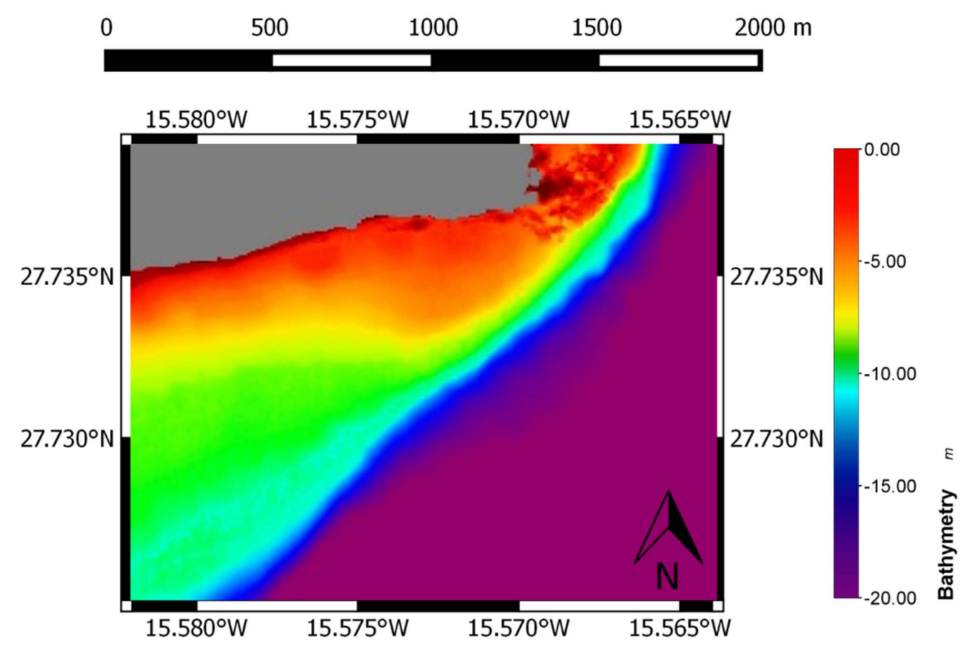

(b)

Figure 10. Maspalomas coastal shallow water bathymetry: (a) Sonar bathymetry; and (b) bathymetry map by using the new deglinting algorithm integrated in the RTE model.

In marine applications, as the water reflectivity is very weak, is where the proper correction of the atmospheric and sun glint becomes more important. In this context, results obtained in the proposed RTM algorithm depend to a certain degree on the WorldView-2 imagery. Limitations in calibration, seasonal solar illumination geometry, viewing effects, atmospheric and sun glint disturbances have a certain impact on the results. Atmospheric correction models, such as $6 \mathrm{~S}$, have proven to provide good results under normal atmospheric conditions. Thus, the sun glint correction algorithm proposed in this paper makes use of the $6 \mathrm{~S}$ normalized direct irradiance model information to analytically obtain the linear parameter $\left(b_{i}\right)$ used in the elimination of the sun glint through the NIR channel. To obtain this expression, the assumption that the refractive index is independent of the wavelength has been considered. Such assumption is systematically used in all sun glint correction algorithms on high resolution images. Anyhow, the minimum refractive index variation of the water with respect to the wavelength of the optical bands allows this approach to introduce very small errors. The proposed method, by eliminating the empirical calculation of the parameter $\left(b_{i}\right)$ using a linear fitting of the reflectivity bands, allows us to remove errors associated with the line regression like outliers generated by the foam, errors in the image resampling or the lack of time synchronization in the image bands. Similarly, the absence of deep water areas without turbidity in the image makes empirical characterization methods ineffective. Finally, integrating sun glint correction algorithm in the radiative transfer modeling is an attempt to solve the main assumption that the reflectivity of water in the NIR channel is negligible. This assumption creates significant errors under very shallow water and high turbidity conditions, so modeling the water reflectivity in the NIR channel allows us mitigate these errors.

\section{Conclusions}

Remote sensing is a useful technology to provide timely and up-to-date information for coastal shallow waters environments. It supplies the spatial and temporal data needed to develop coastal management practices and it enables observations of littoral parameters at greater spatial and finer temporal scales than those allowed through field observation alone. Therefore, it can be a valuable source to monitor water quality parameters, to estimate coastal bathymetry or to retrieve benthic maps.

In this context, high resolution satellite-based imaging systems with spectral bands within the visible spectrum $(400-700 \mathrm{~nm}$ ) can provide the coastal data needed. Unfortunately, remote sensing in water applications presents important challenges mainly due to the complex physical interactions 
of absorption and scattering between water and light, the minimum signal level received at sensor level and the presence of noisy contributions from the atmosphere, solar reflection, foam, turbidity, water column or the seafloor albedo. In this complex scenario, it is critical to apply the appropriate preliminary corrections to successfully obtain the desired marine products.

The use of the atmospheric model 6S has not only provided an atmospheric correction of high accuracy, as validated by in situ data in previous works, but it has also given us insight about some useful information for the sun glint correction algorithm. The utilization of the modeled normalized direct irradiance values has allowed us to obtain the linear coefficient $b_{i}$ relating the glint of the NIR with each of the optical bands analytically. This has permitted us to create a fully automatic algorithm which can be used in scenarios where classic empirical algorithms could not get the $b_{i}$ parameter, as in very shallow waters or inland waters.

Traditional sun glint algorithms are based on the assumption that reflectivity of the water in NIR channels is always negligible. However, that is not the case in very shallow environments or in waters with high concentrations of turbidity. As these are common situations in coastal and in-land waters, significant errors can be introduced. Thus, we have developed a new deglinting methodology based on physical principles for the removal of the sea surface effects from high-resolution imagery.

This paper specifically proposes to integrate the new automatic sun glint removal algorithm in the radiative transfer equation in the pursuit of modeling the contribution of the NIR reflectance of coastal water, which would allow us to eliminate the contribution of the specular NIR reflectance.

Using this radiative model to compute water quality has yielded good results as it considers the physical phenomena of water absorption-backscattering and the relationship between the seafloor albedo, its depth and the water Inherent Optical Properties, which are a function of water quality and the turbidity, CDOM, and chlorophyll-a concentration.

In addition, seafloor albedo and bathymetry maps for the Maspalomas and Corralejo-Lobo Island areas have also been produced, improving previous results using tradition deglinting algorithms.

In summary, a novel Worldview-2 processing methodology has been developed to properly retrieve coastal shallow water parameters. This approach has been validated over a database of in situ measurements collected during field campaigns. Moreover, results have been produced using very high resolution multispectral imagery with different levels of sun glint and for different areas.

Finally, it is important to emphasize that due to the high cost of Worldview-2 images, we have developed a processing approach that must work in an operational environment. Usually, research works are only presented for ideal scenarios, selecting a specific image with clear and calm waters which seldom appear in real situations. However, our aim has been to provide a robust method that achieves acceptable results for a broad range of sea state conditions.

Acknowledgments: This work has been supported by ARTEMISAT (CGL2013-46674-R) and TELECAN MAC/3/C181 (Programa de Cooperación Transnacional Madeira-Azores-Canarias 2007/2013) projects, funded by the Spanish Ministerio de Economía y Competitividad and the European Commission, respectively. We would like to express our special gratitude to the anonymous reviewers for their constructive comments and suggestions.

Author Contributions: Javier Martín, Francisco Eugenio, and Javier Marcello designed the research under the $\mathrm{PhD}$ thesis of Javier Martín. As principal researcher, Javier Martín contributed to algorithm implementation, data preparation, and results generation. Francisco Eugenio, Javier Marcello, and Anabella Medina supported and contributed to all phases of the research. In particular Anabella Medina contributed to data preprocessing and atmospheric correction. All authors contributed to manuscript writing and revisions.

Conflicts of Interest: The authors declare no conflict of interest.

\section{References}

1. Richards, J.A. Remote Sensing Digital Image Analysis; Springer: Berlin, Germany, 2013.

2. Miller, R.L.; del Castillo, C.; McKee, B. Remote Sensing of Coastal Aquatic Environments; Springer: Berlin, Germany, 2005.

3. Abdolrassoul, S.; Mahiny, B.; Turner, J. A comparison of four common atmospheric correction methods. Photogramm. Eng. Remote Sens. 2007, 73, 361-368. 
4. Eugenio, F.; Marcello, J.; Martin, J. High-resolution maps of bathymetry and benthic habitats in shallow-water environments using multispectral remote sensing imagery. IEEE Trans. Geosci. Remote Sens. 2015, 53, 3539-3549. [CrossRef]

5. Kay, S.; Hedley, J.; Lavender, S. Sun glint correction of high and low spatial resolution images of aquatic scenes: A review of methods for visible and near-infrared wavelengths. Remote Sens. 2009, 1, 697-730. [CrossRef]

6. Adler-Golden, S.M.; Acharya, P.A.; Berk, A.; Matthew, M.; Gorodetzky, D. Remote bathymetry of the littoral zone from AVIRIS, LASH and QuickBird imagery. IEEE Trans. Geosci. Remote Sens. 2005, 43, 337-347. [CrossRef]

7. Morel, A. Optical modeling of the upper ocean in relation to its biogenous matter content (case I waters). J. Geophys. Res. Oceans 1988, 93, 10749-10768. [CrossRef]

8. Vermote, E.; Tanré, D.; Deuzé, J.L.; Herman, M.; Morcrette, J.J.; Kotchenova, S.Y. Second Simulation of a Satellite Signal in the Solar Spectrum-Vector (6SV). Available online: http:/ / 6s.ltdri.org/files/tutorial/ 6S_Manual_Part_1.pdf (accessed on 21 December 2015).

9. Svetlana, Y.; Kotchenova, E.; Vermote, F.; Raffaella, M.; Klemm, F.J., Jr. Validation of vector version of $6 \mathrm{~s}$ radiative transfer code for atmospheric correction of satellite data. Part I. Parth radiance. Appl. Opt. 2006, 45, 6762-6774.

10. Mobley, C.D. Hydrolight 4.0 Users Guide; Sequoia Scientific Inc.: Mercer Island, WA, USA, 1998.

11. Sokoletsky, L.G.; Fang, S. Optical closure for remote-sensing reflectance based on accurate radiative transfer approximations: The case of the Changjiang (Yangtze) River Estuary and its adjacent coastal area, China. Int. J. Remote Sens. 2014, 35, 4193-4224. [CrossRef]

12. DigitalGlobe, Inc. Radiometric Use of WorldView-2 Imagery: Technical Note 2010. Available online: http://global.digitalglobe.com/sites/default/files/Radiometric_Use_of_WorldView-2_Imagery $\% 20 \% 281 \%$ 29.pdf (accessed on 1 December 2015).

13. Doxani, G.; Papadopoulou, M.; Lafazani, M.; Tsakiri-Strati, M.; Mavridou, E. Sun Glint Correction of Very High Spatial Resolution Images. Available online: https://www.researchgate.net/publication/ 258843930_Sun_glint_correction_of_very_high_spatial_resolution_images (accessed on 21 December 2015).

14. Cornette, W.M.; Shanks, J. Physically reasonable analytic expression for the single-scattering phase function. Appl. Opt. 1992, 31, 3152-3160. [CrossRef] [PubMed]

15. Cavalli, R.M.; Pignatti, S.; Zappitelli, E. Correction of sun glint effect on MIVIS data of the Sicily campaign in July 2000. Ann. Geophys. 2006, 49. [CrossRef]

16. Cox, C.; Munk, W. Slopes of the Sea Surface Deduced from Photographs of Sun Glitter; Scripps Institution of Oceanography: San Diego, CA, USA, 1956.

17. Garaba, S.P.; Zielinski, O. Methods in reducing surface reflected glint for shipborne above-water remote sensing. J. Eur. Opt. Soc. Rapid Publ. 2013, 8. [CrossRef]

18. Maritorena, S.; Morel, A.; Gentilly, B. Diffuse reflectance of oceanic shallow waters: Influence of water depth and bottom albedo. Limn. Ocean. 1994, 39, 1689-1703. [CrossRef]

19. Zhonping, L. Hyperspectral remote sensing for shallow waters: 2. Deriving bottom depths and water properties by optimization. Appl. Opt. 1999, 38, 3831-3843.

20. Kokhanovsky, A.; Sokoletsky, L.G. Reflection of light from semi-infinite absorbing turbid media. Part 1: Spherical albedo. Color Res. Appl. 2006, 31, 491-497. [CrossRef]

21. Reynolds, R.A.; Stramski, D.; Mitchell, B.G. A chlorophyll-dependent semianalytical reflectance model derived from field measurements of absorption and backscattering coefficients within the Southern Ocean. J. Geophys. Res. 2001, 106, 7125-7138. [CrossRef]

22. Loisel, H.; Stramski, D.; Greg Mitchell, B.; Fell, F.; Fournier-Sicre, V.; Lemasle, B.; Babin, M. Comparison of the ocean inherent optical properties obtained from measurements and inverse modeling. Appl. Opt. 2001, 40, 2384-2397. [CrossRef] [PubMed]

23. Gavin, H.P. The Levenberg-Marquardt Method for Nonlinear Least Squares Curve-Fitting Problems. Available online: http:/ / people.duke.edu/ hpgavin/ce281/lm.pdf (accessed on 30 December 2015). 
24. Camacho, M.A. Depth Analysis of Midway Atoll Using QuickBird Multi-Spectral Imaging over Variable Substrates. Ph.D. Thesis, Naval Postgraduate School, Monterey, CA, USA, 2006.

25. Marcello, J.; Eugenio, F.; Estrada-Allis, S.; Sangrà, P. Segmentation and tracking of anticyclonic eddies during a submarine volcanic eruption using ocean colour imagery. Sensors 2015, 15, 8732-8748. [CrossRef] [PubMed]

(C) 2016 by the authors; licensee MDPI, Basel, Switzerland. This article is an open access article distributed under the terms and conditions of the Creative Commons by Attribution (CC-BY) license (http://creativecommons.org/licenses/by/4.0/). 